\title{
INTERPRETATION OF LAVRENTIEV PHENOMENON BY RELAXATION: THE HIGHER ORDER CASE
}

\author{
MARINO BELLONI
}

ABSTRACT. We consider integral functionals of the calculus of variations of the form

$$
F(u)=\int_{0}^{1} f\left(x, u, u^{\prime}, \ldots, u^{(n)}\right) d x
$$

defined for $u \in W^{n, \infty}(0,1)$, and we show that the relaxed functional $\bar{F}$ with respect to the weak $W_{\text {loc }}^{n, 1}(0,1)$ convergence can be written as

$$
\bar{F}(u)=\int_{0}^{1} f\left(x, u, u^{\prime}, \ldots, u^{(n)}\right) d x+L(u),
$$

where the additional term $L(u)$, the Lavrentiev Gap, is explicitly identified in terms of $F$.

\section{INTRODUCTION}

In 1926 M. Lavrentiev (see [L]) first demonstrated this surprising result: given a variational integral of a two-point Lagrange problem, which is sequentially weakly lower semicontinuous on the admissible class of absolutely continuous functions, its infimum on the dense subclass of $C^{1}$ admissible functions may be strictly greater than its minimum value on the full admissible class. Some years later Manià (see [M]) gave an example of this phenomenon with a polynomial integrand. In recent years there have been additional works by several authors; for further bibliographical references the reader can see for instance [BuM].

In this paper we follow the Buttazzo and Mizel [BuM] approach which consists in studying the Lavrentiev Phenomenon from the point of view of relaxation theory. More precisely let $X$ be a topological space, $Y \subset X$ a dense subset, $F: X \rightarrow[0,+\infty]$ a given functional, and define

$$
\begin{aligned}
\bar{F}_{X} & =\sup \{G: X \rightarrow[0,+\infty]: G \text { l.s.c., } G \leq F \text { on } X\}, \\
\bar{F}_{Y} & =\sup \{G: X \rightarrow[0,+\infty]: G \text { l.s.c., } G \leq F \text { on } Y\}, \\
L(u) & =\left\{\begin{array}{lll}
\bar{F}_{Y}(u)-\bar{F}_{X}(u) & \text { if } \bar{F}_{X}(u)<+\infty, & u \in X . \\
0 & \text { otherwise, }
\end{array}\right.
\end{aligned}
$$

We call this nonnegative functional $L$ (notice that $\bar{F}_{X} \leq \bar{F}_{Y}$ ) the "Lavrentiev Gap" associated to $F, X$ and $Y$. In their paper Buttazzo and Mizel [BuM]

Received by the editors July 13, 1994.

1991 Mathematics Subject Classification. Primary 49J45; Secondary 49K05. 
considered integral functionals of the form

$$
G(u)=\int_{0}^{1} f\left(x, u(x), u^{\prime}(x)\right) d x
$$

with $X=W^{1,1}(0,1)$ and $Y=W^{1,+\infty}(0,1)$, and gave a characterization of $L$ in term of the "Value Function" $V$ (see $\$ 2$ below). Then they obtained an explicit representation of $L$ for a large class of integrands.

In this paper we extend the results of $[\mathrm{BuM}]$ to integral functionals depending on higher order derivatives, of the form

$$
G(u)=\int_{0}^{1} f\left(x, u(x), \ldots, u^{(n)}(x)\right) d x
$$

with $X=W^{n, 1}(0,1)$ and $Y=W^{n,+\infty}(0,1)$. More precisely, in $\S 2$ we obtain a characterization of $L$ in terms of the "Value Function" $V$; in $\S 3$ we provide an explicit representation of $L$ for some integrands which satisfy a "homogeneity condition", and an integrand of Manià type (see [M], [BM1], [BM2]) is analyzed in detail by following this approach. Our results deal with regular integrands (in a sense to be specified), but we want to point out an interesting result involving autonomous second order integrands (see Cheng [C], Cheng and Mizel [CM]) showing the nonoccurrence of the gap phenomenon when the integrand satisfies some continuity assumptions, with an example of a nonvanishing gap when a constraint of the form $\{u \geq 0\}$ is added.

\section{THE REPRESENTATION THEOREM}

Let $\Omega$ be the interval $(0,1)$; we consider the following spaces:

$$
\begin{array}{ll}
W^{n, 1}(0,1) \quad \begin{array}{l}
\text { the space of all functions } u: \Omega \rightarrow \mathbf{R} \text { which are absolutely } \\
\text { continuous together with their }(n-1) \text { derivatives; }
\end{array} \\
W^{n, \infty}[0,1] \quad \begin{array}{l}
\text { the space of all functions } u: \Omega \rightarrow \mathbf{R} \text { which are Lipschitz } \\
\text { continuous together with their }(n-1) \text { derivatives; }
\end{array} \\
\left.\left.W_{\text {loc }}^{n, \infty}\right] 0,1\right] \quad \begin{array}{l}
\text { the space of all functions } u: \Omega \rightarrow \mathbf{R} \text { which are Lipschitz } \\
\text { continuous together with their }(n-1) \text { derivatives on every }
\end{array} \\
\\
\text { interval }[\delta, 1], \text { with } \delta>0 ; \\
\\
\begin{array}{l}
\text { the space of all function } \left.\left.u \in W^{n, 1}(0,1) \cap W_{\text {loc }}^{n, \infty}\right] 0,1\right] \\
\text { such that } u^{(i)}(0)=0 \text { for } i=0, \ldots,(n-1) .
\end{array}
\end{array}
$$

Let $f: \Omega \times \mathbf{R}^{n} \times \mathbf{R} \rightarrow \mathbf{R}$ be a function such that:

(i) $f(x, s, z)$ is of Carathéodory type (i.e. measurable in $X$ and continuous in $(s, z))$;

(ii) $f(x, s, \cdot)$ is convex on $\mathbf{R}$ for every $(x, s) \in \Omega \times \mathbf{R}^{n}$;

(iii) there exists a function $\omega: \Omega \times \mathbf{R} \times \mathbf{R} \rightarrow[0,+\infty[$, with $\omega(x, t, \tau)$ integrable in $x$ and increasing in $t, \tau$, such that

$$
0 \leq f(x, s, z) \leq \omega(x,|s|,|z|) \quad \forall(x, s, z) \in \Omega \times \mathbf{R}^{n} \times \mathbf{R} .
$$


For every $u \in \mathscr{A}_{\infty}$, define

$$
\begin{aligned}
& F(u)=\int_{0}^{1} f\left(x, u, \ldots, u^{(n)}\right) d x \\
& G(u)= \begin{cases}F(u) & \text { if } u \in W^{n, \infty}[0,1] \\
+\infty & \text { otherwise }\end{cases}
\end{aligned}
$$

and denote by $\bar{G}$ the functional

$$
\bar{G}=\max \left\{H: \mathscr{A}_{\infty} \rightarrow[0,+\infty]: H \text { seq. w- } W_{\text {loc }}^{n, 1} \text {-1.s.c., } H \leq G\right\} .
$$

Our goal is to give a representation formula for $\bar{G}$ over $\mathscr{A}_{\infty}$.

Since $F$ is sequentially weakly lower semicontinuous on $W_{\text {loc }}^{n, 1}(0,1)$ (briefly seq. $\mathrm{w}-W_{\text {loc }}^{n, 1}(0,1)$-l.s.c.) (see [B]), we have

$$
\bar{G}(u) \geq F(u) \quad \forall u \in \mathscr{A}_{\infty},
$$

and then

$$
\bar{G}(u)=F(u)+L(u) \quad \forall u \in \mathscr{A}_{\infty}
$$

for a suitable functional $L \geq 0$. We call the functional $L$ the "Lavrentiev Gap" relative to $G$ over the space $\mathscr{A}_{\infty}$. Obviously we have that $\bar{G} \leq G$. Then

$$
\bar{G}(u)=F(u) \quad \forall u \in W^{n, \infty}[0,1] ;
$$

i.e. $L(u)=0$ for every $u \in W^{n, \infty}[0,1]$. In order to identify the functional $L$ we introduce the "Value Function" $V(x, s)$ defined for every $(x, s) \in \Omega \times \mathbf{R}^{n}$ by:

$$
\begin{array}{r}
V(x, s)=\inf \left\{\int_{0}^{x} f\left(t, u, \ldots, u^{(n)}\right) d t: u \in W^{n, \infty}[0,1], u^{(i)}(0)=0,\right. \\
\left.u^{(i)}(x)=s_{i}, i=0, \ldots,(n-1)\right\}
\end{array}
$$

and its lower semicontinuous envelope with respect to $s=\left(s_{0}, \ldots, s_{(n-1)}\right)$, given by

$$
W(x, s)=\liminf _{\xi \rightarrow s} V(x, \xi) .
$$

We now state a representation result for the Lavrentiev Gap $L$.

Theorem 2.1. If the integrand $f(x, s, z)$ satisfies the hypotheses above, then

$$
L(u)=\liminf _{x \rightarrow 0^{+}} W\left(x, u(x), \ldots, u^{(n-1)}(x)\right) \text { for every } u \in \mathscr{A}_{\infty} .
$$

In order to achieve the proof of Theorem 2.1 we need some lemmas. For the sake of simplicity in the following we set

$$
M(u)=\liminf _{x \rightarrow 0^{+}} W\left(x, u(x), \ldots, u^{(n-1)}(x)\right)
$$

and, when no confusion is possible, we use the notation $\bar{u}(x)$ to indicate the vector $\left(u^{(i)}(x)\right)_{i=0}^{n-1}$. 
Lemma 2.2. Take $u, u_{h} \in \mathscr{A}_{\infty}$ with $u_{h} \in W^{n, \infty}[0,1]$ and assume that $u_{h} \rightarrow u$ weakly in $W_{\mathrm{loc}}^{n, 1}(0,1)$. Then

$$
F(u)+M(u) \leq \liminf _{h \rightarrow+\infty} G\left(u_{h}\right) .
$$

Proof. Take $\delta>0$; for every $h \in \mathbf{N}$, by the definitions of $V(x, s)$ and $W(x, s)$ we get

$$
\begin{aligned}
G\left(u_{h}\right) & =\int_{0}^{\delta} f\left(x, u_{h}, \ldots, u_{h}^{(n)}\right) d x+\int_{\delta}^{1} f\left(x, u_{h}, \ldots, u_{h}^{(n)}\right) d x \\
& \geq V\left(\delta, \bar{u}_{h}(\delta)\right)+\int_{\delta}^{1} f\left(x, u_{h}, \ldots, u_{h}^{n}\right) d x \\
& \geq W\left(\delta, \bar{u}_{h}(\delta)\right)+\int_{\delta}^{1} f\left(x, u_{h}, \ldots, u_{h}^{(n)}\right) d x .
\end{aligned}
$$

As $h \rightarrow+\infty$, taking into account that $W$ is seq. $w-W_{\text {loc }}^{n, 1}-1 . s . c$. and that the assumptions on the integrand $f$ provide the seq. $\mathrm{w}-W_{\mathrm{loc}}^{n, 1}-1$.s.c. of the integral term, we get

$$
\begin{aligned}
\liminf _{h \rightarrow+\infty} G\left(u_{h}\right) & \geq \liminf _{h \rightarrow+\infty}\left[W\left(\delta, \bar{u}_{h}(\delta)\right)+\int_{\delta}^{1} f\left(x, u_{h}, \ldots, u_{h}^{(n)}\right) d x\right] \\
& \geq W(\delta, \bar{u}(\delta))+\int_{\delta}^{1} f\left(x, u, \ldots, u^{(n)}\right) d x .
\end{aligned}
$$

Finally, as $\delta \rightarrow 0$ we obtain

$$
\begin{aligned}
\liminf _{h \rightarrow+\infty} G\left(u_{h}\right) & \geq \liminf _{\delta \rightarrow 0}\left[W(\delta, \bar{u}(\delta))+\int_{\delta}^{1} f\left(x, u, \ldots, u^{(n)}\right) d x\right] \\
& \geq M(u)+\int_{0}^{1} f\left(x, u, \ldots, u^{(n)}\right) d x \\
& =M(u)+F(u),
\end{aligned}
$$

and the lemma is proved.

Lemma 2.3. The functional $F+M$ is seq. $w$-W $W_{\text {loc }}^{n, 1}$-l.s.c. on $\mathscr{A}_{\infty}$.

Proof. Taking $u, u_{h} \in \mathscr{A}_{\infty}$ with $u_{h} \rightarrow u$ weakly in $W_{\text {loc }}^{n, 1}$, we have to show that

$$
F(u)+M(u) \leq \liminf _{h \rightarrow+\infty}\left[F\left(u_{h}\right)+M\left(u_{h}\right)\right] .
$$

Assume that the right-hand side is finite (otherwise there is nothing to prove), and consider a sequence $\left(x_{h}\right)$ in $\Omega$ with $x_{h} \rightarrow 0$ such that

$$
W\left(x_{h}, \bar{u}_{h}\left(x_{h}\right)\right) \geq M\left(u_{h}\right)+\frac{1}{h} \quad \forall h \in \mathbf{N} .
$$

It is now possible to find a sequence $\left(s_{h}\right)$ in $\mathbf{R}^{n}$, with $s_{h} \rightarrow 0$ such that

$$
\begin{gathered}
\left|s_{h}-\bar{u}_{h}\left(x_{h}\right)\right| \leq \frac{1}{h} ; \\
V\left(x_{h}, s_{h}\right) \leq W\left(x_{h}, \bar{u}_{h}\left(x_{h}\right)\right)+\frac{1}{h} .
\end{gathered}
$$


Moreover, denoting by $P_{n-1}$ the polynomial of degree $n-1$ such that $\bar{P}_{n-1}\left(x_{h}\right)$ $=s_{h}-\bar{u}_{h}\left(x_{h}\right)$, it is easy to see that, since $f$ is of Carathéodory type, the sequence $\left(s_{h}\right)$ can be taken such that

$$
\int_{x_{h}}^{1} f\left(x, \bar{u}_{h}+\bar{P}_{n-1}, u_{h}^{(n)}\right) d x \leq \int_{x_{h}}^{1} f\left(x, \bar{u}_{h}, u_{h}^{(n)}\right) d x+\frac{1}{h} .
$$

Finally, let $v_{h} \in W^{n, \infty}\left[0, x_{h}\right]$ be such that $\bar{v}_{h}(0)=0, \bar{v}_{h}\left(x_{h}\right)=s_{h}$ and

$$
\int_{0}^{x_{h}} f\left(x, \bar{v}_{h}, v_{h}^{(n)}\right) d x \leq V\left(x_{h}, s_{h}\right)+\frac{1}{h} .
$$

Setting

$$
w_{h}(x)= \begin{cases}u_{h}(x)+P_{n-1}(x) & \text { if } x>x_{h}, \\ v_{h}(x) & \text { if } 0 \leq x \leq x_{h},\end{cases}
$$

we have $w_{h} \in W^{n, \infty}[0,1], \bar{w}_{h}(0)=0$, and

$$
w_{h} \underset{h \rightarrow+\infty}{\longrightarrow} u \mathrm{w}-W_{\mathrm{loc}}^{n-1}(0,1) .
$$

Therefore, by using Lemma 2.2 and $(2.1)-(2.6)$, we obtain

$$
\begin{aligned}
F(u) & +M(u) \leq \liminf _{h \rightarrow+\infty} F\left(w_{h}\right) \\
& =\liminf _{h \rightarrow+\infty}\left[\int_{0}^{x_{h}} f\left(x, \bar{v}_{h}, v_{h}^{(n)}\right) d x+\int_{x_{h}}^{1} f\left(x, \bar{u}_{h}+\bar{P}_{n-1}\left(x_{h}\right), u_{h}^{(n)}\right) d x\right] \\
& \leq \liminf _{h \rightarrow+\infty}\left[\left(V\left(x_{h}, s_{h}\right)+\frac{1}{h}\right)+\left(\int_{x_{h}}^{1} f\left(x, \bar{u}_{h}, u_{h}^{(n)}\right) d x+\frac{1}{h}\right)\right] \\
& \leq \liminf _{h \rightarrow+\infty}\left[\left(W\left(x_{h}, \bar{u}_{h}\left(x_{h}\right)\right)+\frac{1}{h}\right)+F\left(u_{h}\right)+\frac{2}{h}\right] \\
& \leq \liminf _{h \rightarrow+\infty}\left[\left(M\left(u_{h}\right)+\frac{1}{h}\right)+F\left(u_{h}\right)+\frac{3}{h}\right] \\
& =\liminf _{h \rightarrow+\infty}\left[M\left(u_{h}\right)+F\left(u_{h}\right)\right],
\end{aligned}
$$

and the lemma is proved.

Proof of Theorem 2.1. It is easy to see that

$$
M(u)=0 \quad \text { for every } u \in \mathscr{A}_{\infty} \cap W^{n, \infty}[0,1] ;
$$

hence we have $F+M \leq G$ on $\mathscr{A}_{\infty}$. By Lemma 2.3 we have $F+M \leq \bar{G}$ on $\mathscr{A}_{\infty}$, so it remains to prove that

$$
\bar{G} \leq F(u)+M(u) \text { for every } u \in \mathscr{A}_{\infty} .
$$

To this aim, fix $u \in \mathscr{A}_{\infty}$ and take a sequence $\left(x_{h}\right)$ in $\Omega, x_{h} \rightarrow 0$, such that

$$
M(u)=\lim _{h \rightarrow+\infty} W\left(x_{h}, \bar{u}\left(x_{h}\right)\right) .
$$


By definition of $W$ and the assumptions on the integrand $f$ we may find a sequence $\left(s_{h}\right)$ in $\mathbf{R}^{n}, s_{h} \rightarrow 0$, such that for every $h \in \mathbf{N}$

$$
\begin{gathered}
\left|s_{h}-\bar{u}\left(x_{h}\right)\right| \leq \frac{1}{h}, \\
V\left(x_{h}, s_{h}\right) \leq W\left(x_{h}, \bar{u}\left(x_{h}\right)\right)+\frac{1}{h}, \\
\int_{x_{h}}^{1} f\left(x, \bar{u}+\bar{P}_{n-1}, u^{(n)}\right) d x \leq \int_{x_{h}}^{1} f\left(x, \bar{u}, u^{(n)}\right) d x+\frac{1}{h},
\end{gathered}
$$

where $P_{n-1}$ is as in the proof of Lemma 2.3. Finally, let $v_{h} \in W^{n, \infty}\left[0, x_{h}\right]$ be a sequence such that $\bar{v}_{h}(0)=0, \bar{v}_{h}\left(x_{h}\right)=s_{h}$ and

$$
\int_{0}^{x_{h}} f\left(x, \bar{v}_{h}, v_{h}^{(n)}\right) d x \leq V\left(x_{h}, s_{h}\right)+\frac{1}{h} .
$$

As in the proof of Lemma 2.3, we define the sequence

$$
w_{h}(x)= \begin{cases}u_{h}(x)+P_{n-1}(x) & \text { if } x>x_{h}, \\ v_{h}(x) & \text { if } 0 \leq x \leq x_{h} .\end{cases}
$$

Then $w_{h} \in W^{n, \infty}[0,1], \bar{w}_{h}(0)=0$ and

$$
w_{h} \underset{h \rightarrow+\infty}{\longrightarrow} u \text { strongly } W_{\text {loc }}^{n, 1}(0,1) .
$$

Hence, by using (2.7)-(2.11), we obtain

$$
\begin{aligned}
\bar{G}(u) & \leq \liminf _{h \rightarrow+\infty} G\left(w_{h}\right) \\
& =\liminf _{h \rightarrow+\infty}\left[\int_{0}^{x_{h}} f\left(x, \bar{v}_{h}, v_{h}^{(n)}\right) d x+\int_{x_{h}}^{1} f\left(x, \bar{u}+\bar{P}_{n-1}\left(x_{h}\right), u^{(n)}\right) d x\right] \\
& \leq \liminf _{h \rightarrow+\infty}\left[\left(V\left(x_{h}, s_{h}\right)+\frac{1}{h}\right)+\left(\int_{x_{h}}^{1} f\left(x, \bar{u}, u^{(n)}\right) d x+\frac{1}{h}\right)\right] \\
& \leq \liminf _{h \rightarrow+\infty}\left[\left(W\left(x_{h}, \bar{u}\left(x_{h}\right)\right)+\frac{1}{h}\right)+F(u)+\frac{2}{h}\right] \\
& =M(u)+F(u) ;
\end{aligned}
$$

so $M=L$, and the theorem is completely proved.

Remark 2.4. Fix a subset $\beta$ of $\{0,1, \ldots, n-1\}$ and consider the class $\mathscr{A}_{\infty}^{\beta}$ of all functions $\left.u \in W^{n, 1}(0,1) \cap W_{\mathrm{loc}}^{n, \infty} \mathrm{j} 0,1\right]$ such that $u^{(i)}(0)=0$ for $i \in \beta$. We denote by $\bar{G}_{\beta}$ the functional

$$
\bar{G}_{\beta}=\max \left\{H: \mathscr{A}_{\infty}^{\beta} \rightarrow[0,+\infty]: H \text { seq. w- } W_{\text {loc }}^{n, 1}-1 \text {.s.c., } H \leq G\right\} .
$$

As in the previous case, we have

$$
\bar{G}_{\beta}(u)=F(u)+L_{\beta}(u) \quad \forall u \in \mathscr{A}_{\infty}
$$

for a suitable functional $L_{\beta} \geq 0$, the "Lavrentiev Gap" relative to $G$ over the space $\mathscr{A}_{\infty}^{\beta}$. In order to identify the functional $L_{\beta}$ we introduce the Value 
Function $V_{\beta}(x, s)$ defined for every $(x, s) \in \Omega \times \mathbf{R}^{k}$ by:

$$
\begin{array}{r}
V_{\beta}(x, s)=\inf \left\{\int_{0}^{x} f\left(t, u, \ldots, u^{(n)}\right) d t: u \in W^{n, \infty}[0,1], u^{\left(i_{j}\right)}(0)=0,\right. \\
\left.u^{\left(i_{j}\right)}(x)=s_{i_{j}}, j=0,1, \ldots, k-1\right\}
\end{array}
$$

and its lower semicontinuous envelope with respect to $s=\left(s_{i_{0}}, \ldots, s_{i_{k-1}}\right)$, given by

$$
W_{\beta}(x, s)=\liminf _{\xi \rightarrow s} V_{\beta}(x, \xi) .
$$

By repeating step by step the proof of Theorem 2.1, we obtain the following result:

Theorem 2.5. If the integrand $f(x, s, z)$ satisfies the assumptions of Theorem 2.1 , then

$$
L_{\beta}(u)=\liminf _{x \rightarrow 0^{+}} W_{\beta}\left(x, u^{\left(i_{0}\right)}(x), \ldots, u^{\left(i_{k-1}\right)}(x)\right) \quad \text { for every } u \in \mathscr{A}_{\infty}^{\beta} .
$$

Note that the polynomial $P_{n-1}$ may be chosen, in this case, such that

$$
P_{n-1}^{(r)}= \begin{cases}u_{h}^{(r)}\left(x_{h}\right)-\left(s_{h}\right)_{r} & \text { if } r \in \beta \\ 0 & \text { otherwise }\end{cases}
$$

\section{SOME EXAMPLES}

In this section we give an explicit representation formula for a class of second order integrands $f$ (we mean that $f$ is a function depending on $x, u, u^{\prime}, u^{\prime \prime}$ ). We introduce the so-called "invariance property" for second order integrands (analogous to the one introduced in [HM1] for first order integrands, and to the one of $[\mathrm{CM}]$ for second order autonomous integrands):

there exists $\gamma \in] 1,2[$ such that for every $t>0$ and $(x, s, z, w)$

$\in \Omega \times \mathbf{R}^{3} t f\left(t x, t^{\gamma} s, t^{\gamma-1} z, t^{\gamma-2} w\right)=f(x, s, z, w)$.

We want to analyze a class of second order integrands $f(x, s, z, w)$ that satisfies this invariance property only in an asymptotic sense near the relevant singular abscissa. Let us take $\delta>1, \tau \in[1, \delta[$; we suppose the integrand $f: \Omega \times \mathbf{R} \times \mathbf{R} \rightarrow \mathbf{R}$ has the form

$$
f(x, s, z, w)=x^{\tau-1} a(x, s) b(x, z)|w|^{\delta},
$$

with $a(x, s), b(x, z)$ nonnegative, continuous functions such that, setting $\gamma=$ $2-\frac{\tau}{\delta}$, for every $y \in \Omega$ the functions $m_{y}, n_{y}, M_{y}, N_{y}: \Omega \rightarrow \mathbf{R}$ defined by

$$
\begin{array}{ll}
m_{y}(s)=\inf \left\{a\left(x, x^{\gamma} s\right): x \leq y\right\}, & n_{y}(s)=\inf \left\{b\left(x, x^{\gamma-1} s\right): x \leq y\right\}, \\
M_{y}(s)=\sup \left\{a\left(x, x^{\gamma} s\right): x \leq y\right\}, & N_{y}(s)=\sup \left\{b\left(x, x^{\gamma-1} s\right): x \leq y\right\}
\end{array}
$$

are locally bounded. Take now $x, y \in \Omega, x \leq y$, and consider the following functionals:

$$
\begin{aligned}
F_{x}(u) & =\int_{0}^{x} f\left(t, u, u^{\prime}, u^{\prime \prime}\right) d t \\
F_{*, x, y}(u) & =\int_{0}^{x} t^{\tau-1} m_{y}\left(t^{-\gamma} u\right) n_{y}\left(t^{1-\gamma} u^{\prime}\right)\left|u^{\prime \prime}\right|^{\delta} d t \\
F_{x, y}^{*}(u) & =\int_{0}^{x} t^{\tau-1} M_{y}\left(t^{-\gamma} u\right) N_{y}\left(t^{1-\gamma} u^{\prime}\right)\left|u^{\prime \prime}\right|^{\delta} d t .
\end{aligned}
$$


We suppose that there exists $\bar{y} \in \Omega$ such that, for every $x \in\{x \in \Omega: x \leq \bar{y}\}$, we have

$$
F_{x, \bar{y}}^{*}(u)<+\infty \text { whenever } F_{x}(u)<+\infty .
$$

Obviously for every $x, y \in \Omega$ with $x \leq y$

$$
F_{*, x, y}(u) \leq F_{x}(u) \leq F_{x, y}^{*}(u) \quad \forall u \in \mathscr{A}_{\infty}^{1} ;
$$

then for every $x \in \Omega$

$$
\sup _{\substack{y \in \Omega \\ y \leq \bar{y}}} F_{*, x, y}(u) \leq F_{x}(u) \leq \inf _{\substack{y \in \Omega \\ y \leq \bar{y}}} F_{x, y}^{*}(u) \quad \forall u \in \mathscr{A}_{\infty}^{1},
$$

say

$$
\lim _{y \rightarrow 0^{+}} F_{*, x, y}(u) \leq F_{x}(u) \leq \lim _{y \rightarrow 0^{+}} F_{x, y}^{*}(u) \quad \forall u \in \mathscr{A}_{\infty}^{1} .
$$

Define, for $y \leq \bar{y}$,

$$
\begin{array}{cl}
\lim _{y \rightarrow 0^{+}} m_{y}(s)=m_{0}(s), & \lim _{y \rightarrow 0^{+}} M_{y}(s)=M_{0}(s), \\
\lim _{y \rightarrow 0^{+}} n_{y}(s)=n_{0}(s), & \lim _{y \rightarrow 0^{+}} N_{y}(s)=N_{0}(s) ;
\end{array}
$$

by the assumptions (3.1) we apply the Monotone and Lebesgue Convergence Theorems to (3.2) obtaining

$$
F_{0, x}(u) \leq F_{x}(u) \leq F_{x}^{0}(u) \quad \forall u \in \mathscr{A}_{\infty}^{1}
$$

where

$$
\begin{aligned}
F_{0, x}(u) & =\int_{0}^{x} t^{\tau-1} m_{0}\left(t^{-\gamma} u\right) n_{0}\left(t^{1-\gamma} u^{\prime}\right)\left|u^{\prime \prime}\right|^{\delta} d t \\
F_{x}^{0}(u) & =\int_{0}^{x} t^{\tau-1} M_{0}\left(t^{-\gamma} u\right) N_{0}\left(t^{1-\gamma} u^{\prime}\right)\left|u^{\prime \prime}\right|^{\delta} d t .
\end{aligned}
$$

We suppose also that $m_{0}(s)=P \leq Q=M_{0}(s)$, with $P, Q \in[0,+\infty[$.

Theorem 3.1. Under the previous assumptions, for every

$$
\left.\left.u \in \mathscr{A}_{\infty}^{1}=\left\{u \in W^{2,1}(0,1) \cap W_{\mathrm{loc}}^{2, \infty}\right] 0,1\right]: u^{\prime}(0)=0\right\}
$$

we have

$$
\begin{aligned}
& \left.P \delta k^{\delta-1}\left|\int_{0}^{\lim \inf _{x \rightarrow 0} u^{\prime}(x) x^{1-\gamma}} n_{0}(\xi)\right| \xi\right|^{\delta-1 d \xi} \mid \\
& \quad \leq L(u) \leq\left. Q \delta k^{\delta-1}\left|\int_{0}^{\lim \inf _{x \rightarrow 0} u^{\prime}(x) x^{1-\gamma}} N_{0}(\xi)\right| \xi\right|^{\delta-1} d \xi \mid
\end{aligned}
$$

where $k=\frac{\delta(\gamma-1)}{\delta-1}$.

In order to achieve the proof of Theorem 3.1, we need a lemma. 
Lemma 3.2. Let $h(Z)$ be the solution of the minimum problem

$$
\inf \left\{G(u): u \in W^{2, \infty}(0,1), u^{\prime}(0)=0, u^{\prime}(1)=Z\right\}
$$

where

$$
G(u)=\int_{0}^{1} x^{\tau-1} n\left(x^{1-\gamma} u^{\prime}(x)\right)\left|u^{\prime \prime}(x)\right|^{\delta} d x
$$

We have

$$
h(Z)=\left.\delta k^{\delta-1}\left|\int_{0}^{Z} n(\xi)\right| \xi\right|^{\delta-1} d \xi \mid,
$$

where $k=\frac{\delta(\gamma-1)}{\delta-1}$ and the function $h(Z)$ is the solution of the equation

$$
\left\{\begin{array}{l}
(\gamma-1) Z h^{\prime}(Z)=\sup \left\{Q h^{\prime}(Z)-n(Z)|Q|^{\delta}: Q \in \mathbf{R}\right\}, \\
h(0)=0
\end{array}\right.
$$

Proof. By explicitly carrying out the maximization, the equation (3.5) becomes

$$
h^{\prime}(Z)=\delta k^{\delta-1} n(Z)|Z|^{\delta-2}
$$

and by direct integration

$$
h(Z)=\left.\delta k^{\delta-1}\left|\int_{0}^{Z} n(\xi)\right| \xi\right|^{\delta-1} d \xi \mid .
$$

Let us take $u \in \mathscr{A}(x, z)=\left\{u \in W^{2, \infty}: u^{\prime}(0)=0, u^{\prime}(x)=z\right\}$; from (3.5), setting $Z(t)=t^{1-\gamma} u^{\prime}(t)$ and $Q(t)=t^{2-\gamma} u^{\prime \prime}(t)$ we obtain

$$
(\gamma-1) Z(t) h^{\prime}(Z(t)) \geq|Q(t)| h^{\prime}(Z(t))-n(Z(t))|Q(t)|^{\delta} .
$$

Then

$$
\begin{aligned}
t^{-1} n(Z(t))|Q(t)|^{\delta} & \geq t^{-1} h^{\prime}(Z(t))[Q(t)+(1-\gamma) Z(t)] \\
& =h^{\prime}(Z(t)) Z^{\prime}(t)=(h \circ Z)^{\prime}(t)
\end{aligned}
$$

(for the last equality see $[\mathrm{MM}]$ ). Integrating on $] 0, x[$ yields

$$
\begin{aligned}
I(u) & =\int_{0}^{x} t^{\tau-1} n\left(t^{1-\gamma} u^{\prime}(t)\right)\left|u^{\prime \prime}(t)\right|^{\delta} d t \\
& =\int_{0}^{x} t^{-1} n\left(t^{1-\gamma} u^{\prime}(t)\right)\left|t^{\tau / \delta} u^{\prime \prime}(t)\right|^{\delta} d t \\
& =\int_{0}^{x} t^{-1} n(Z(t))|Q(t)|^{\delta} d t \\
& \geq \int_{0}^{x}(h \circ Z)^{\prime}(t) d t \\
& =h(Z(x))-\lim _{t \rightarrow 0^{+}} h(Z(t)) \\
& =h(Z(x))
\end{aligned}
$$

(in fact $u \in W^{2, \infty}[0, x]$ with $u^{\prime}(0)=0$ implies

$$
\lim _{t \rightarrow 0^{+}} t^{1-\gamma} u^{\prime}(t)=0 \quad \forall \gamma \in[1,2[,
$$

and hence $\left.\lim _{t \rightarrow 0^{+}} h(Z(t))=0\right)$. It follows that

$$
W(x, z)=\inf \{I(u): u \in \mathscr{A}(x, z)\} \geq h\left(x^{1-\gamma} z\right)=h(Z) \text {. }
$$


Consider now the sequence $\left(u_{\varepsilon}\right) \subset \mathscr{A}(x, z)$ defined by

$$
u_{\varepsilon}(0)=0, \quad u_{\varepsilon}^{\prime}(t)= \begin{cases}\left(\frac{t}{x}\right)^{k} z & \text { if } t \geq \varepsilon, \\ t \frac{\varepsilon^{k-1}}{x^{k}} z & \text { if } t<\varepsilon .\end{cases}
$$

Taking $\varepsilon$ sufficiently small we have

$$
W(x, z) \leq I\left(u_{\varepsilon}\right)=\int_{0}^{\varepsilon} t^{\tau-1} n\left(t^{1-\gamma} u_{\varepsilon}^{\prime}\right)\left|u_{\varepsilon}^{\prime \prime}\right|^{\delta} d t+\int_{\varepsilon}^{x} t^{\tau-1} n\left(t^{1-\gamma} u_{0}^{\prime}\right)\left|u_{0}^{\prime \prime}\right|^{\delta} d t,
$$

where $u_{0}^{\prime}(t)=\left(\frac{t}{x}\right)^{k}, u_{0}(0)=0$; passing to the limit for $\varepsilon \rightarrow 0$ the first integral tends to 0 , and hence

$$
W(x, s) \leq I\left(u_{0}\right) .
$$

At this point we can easily verify that $I\left(u_{0}\right)=h\left(x^{1-\gamma} z\right)$, and the proof of the lemma is then complete.

Proof of Theorem 3.1. We fix $u \in \mathscr{A}_{\infty}^{1}$; by Theorem 2.5

$$
L_{1}(u)=\liminf _{x \rightarrow 0} W_{1}\left(x, u^{\prime}(x)\right)
$$

where $W_{1}(x, z)=\liminf _{q \rightarrow z} V_{1}(x, q)$ and

$$
\begin{aligned}
V_{1}(x, z) & =\inf \left\{F_{x}(u): u \in W^{2, \infty}(0,1), u^{\prime}(0)=0, u^{\prime}(x)=z\right\} \\
& =\inf \left\{F_{x}(u): u \in \mathscr{A}(x, z)\right\}
\end{aligned}
$$

where $\mathscr{A}(x, z)=\left\{u \in W^{2}, \infty(0, x): u^{\prime}(0)=0, u^{\prime}(x)=z\right\}$.

Let us introduce the Value Functions relative to the functionals $F_{0, x}, F_{x}^{0}$ given by

$$
\begin{aligned}
V_{0}(x, z) & =\inf \left\{F_{0, x}(u): u \in \mathscr{A}(x, z)\right\}, \\
V^{0}(x, z) & =\inf \left\{F_{x}^{0}(u): u \in \mathscr{A}(x, z)\right\}
\end{aligned}
$$

obviously, for every $x \in \Omega$ and for every $z \in \mathbf{R}$, we have by (3.3)

$$
V_{0}(x, z) \leq V_{1}(x, z) \leq V^{0}(x, z) .
$$

Setting $S=s x^{-\gamma}, Z=z x^{1-\gamma}$ and

$$
\begin{aligned}
G_{0}(u) & =P \int_{0}^{1} t^{\tau-1} n_{0}\left(t^{1-\gamma} u^{\prime}\right)\left|u^{\prime \prime}\right|^{\delta} d t, \\
G^{0}(u) & =Q \int_{0}^{1} t^{\tau-1} N_{0}\left(t^{1-\gamma} u^{\prime}\right)\left|u^{\prime \prime}\right|^{\delta} d t, \\
\mathscr{A}(Z) & =\left\{u \in W^{2, \infty}(0,1): u^{\prime}(0)=0, u^{\prime}(1)=Z\right\},
\end{aligned}
$$

by the change of variable $t=x y$ we get

$$
\begin{gathered}
V_{0}(x, z)=H_{0}(Z)=\inf \left\{G_{0}(u): u \in \mathscr{A}(Z)\right\}, \\
V^{0}(x, z)=H^{0}(Z)=\inf \left\{G^{0}(u): u \in \mathscr{A}(Z)\right\},
\end{gathered}
$$

so that inequalities (3.8) become

$$
H_{0}(Z) \leq V_{1}(x, z) \leq H^{0}(Z)
$$

for every $x \in \Omega$ and for every $z \in \mathbf{R}$. 
By Lemma 3.2 we have that $H_{0}(Z)$ and $H^{0}(Z)$ are given by

$$
\begin{aligned}
& H_{0}(Z)=\left.P \delta k^{\delta-1}\left|\int_{0}^{Z} n_{0}(\xi)\right| \xi\right|^{\delta-1} d \xi \mid \\
& H^{0}(Z)=\left.Q \delta k^{\delta-1}\left|\int_{0}^{Z} N_{0}(\xi)\right| \xi\right|^{\delta-1} d \xi \mid
\end{aligned}
$$

and inserting (3.10) into (3.9) we obtain the inequality (3.4), that is the thesis.

Example 3.3. Consider the functional

$$
F(u)=\int_{0}^{1} f\left(x, u(x), u^{\prime}(x), u^{\prime \prime}(x)\right) d x,
$$

where the integrand $f$ has the following form, with $1<p<2$ and $0<q<1$,

$$
\begin{aligned}
f(x, s, z, w) & =\left(s-x^{p}\right)^{2}\left(z-x^{q}\right)^{2}|w|^{\delta} \\
& =x^{2(p+q)}\left(s x^{-p}-1\right)^{2}\left(z x^{-q}-1\right)^{2}|w|^{\delta} \\
& =x^{2(p+q)} a(x, s) b(x, z)|w|^{\delta},
\end{aligned}
$$

where we set

$$
\begin{aligned}
& a(x, s)=\left(s x^{-p}-1\right)^{2}, \\
& b(x, z)=\left(z x^{-q}-1\right)^{2} .
\end{aligned}
$$

If $\delta \leq 1$ we can easily verify that the Lavrentiev Gap $L(u)$ is identically equal to 0 : for every $u \in W^{2,1}(0,1)$ with $u^{\prime}(0)=0$ we construct the sequence in $W^{2, \infty}$

$$
\begin{aligned}
& u_{\varepsilon}^{\prime}(x)= \begin{cases}u^{\prime}(x), & \text { if } x_{\varepsilon} \leq x, \\
\frac{u^{\prime}\left(x_{\varepsilon}\right)}{x_{\varepsilon}} x, & \text { if } 0 \leq x \leq x_{\varepsilon},\end{cases} \\
& u_{\varepsilon}(0)=u(0),
\end{aligned}
$$

where $x_{\varepsilon} \in[0,1]$ is a sequence with limit 0 as $\varepsilon \rightarrow 0$, and we verify that $F\left(u_{\varepsilon}\right) \rightarrow F(u)$ as $\varepsilon \rightarrow 0$. Here, for simplicity, we restrict our attention to the case $\delta>\frac{1+2(p+q)}{2-p}$. With the notation above

$$
\tau=1+2(p+q), \quad \gamma=2-\frac{\tau}{\delta}=2-\frac{1+2(p+q)}{\delta} .
$$

This integrand $f$ has as "zero cost curves" the functions $z_{1}(x)=x^{p}, z_{2}(x)=$ $(q+1)^{-1} x^{q+1}$; by the assumption on $p$ and $q$ we obtain $z_{1}(x), z_{2}(x) \in$ $W^{2,1}(0,1) \backslash W^{2, \infty}[0,1]$.

When $\delta>\frac{1+2(p+q)}{1-q}$, we have $\gamma>p, \gamma>q$ and then

$$
m_{0}(s)=n_{0}(s)=M_{0}(s)=N_{0}(s)=1 ;
$$

hence for every fixed $u \in \mathscr{A}_{\infty}^{1}$ we obtain

$$
L_{1}(u)=k^{\delta-1} \liminf _{x \rightarrow 0^{+}}\left|\frac{u^{\prime}(x)}{x^{\gamma-1}}\right|^{\delta}
$$

this functional is not identically equal to 0 : for instance, $L_{1}\left(x^{p}\right)=+\infty$ and $L_{1}\left((q+1)^{-1} x^{q+1}\right)=+\infty$. 
When $\delta=\frac{1+2(p+q)}{1-q}$, by a computation similar to the previous case, we obtain

$$
m_{0}(s)=M_{0}(s)=1, \quad n_{0}(s)=N_{0}(s)=(s-1)^{2} .
$$

Then, for every fixed $u \in \mathscr{A}_{\infty}^{1}$ we have

$$
L_{1}(u)=\left.\delta k^{\delta-1}\left|\liminf _{x \rightarrow 0^{+}} \int_{0}^{u^{\prime}(x) x^{1-\gamma}}(\xi-1)^{2}\right| \xi\right|^{\delta-1} d \xi \mid
$$

also in this case this functional is not identically equal to 0 : for instance $L_{1}\left(x^{p}\right)=+\infty$, while $L_{1}\left((q+1)^{-1} x^{q+1}\right)=2 k^{\delta-1} /(\delta+1)(\delta+2)$.

Finally, when $\delta<\frac{1+2(p+q)}{1-q}$, Theorem 3.1 does not apply because the functions $n_{y}, N_{y}$ are not locally bounded. However it is possible to show that in this case the gap phenomenon does not occur (see [Be]): for every $u \in \mathscr{A}_{W^{2,1}}^{1}$ we construct $u_{\varepsilon}$ in $W^{2, \infty}(0,1)$ by $(3.11)$ and we prove that, if $F(u)<+\infty$, then $F\left(u_{\varepsilon}\right) \rightarrow F(u)$ as $\varepsilon \rightarrow 0$, i.e.

$$
\int_{0}^{1} f\left(x, u, u^{\prime}, u^{\prime \prime}\right) d x<+\infty \Rightarrow L(u)=0 .
$$

\section{ACKNOWLEDGMENTS}

I thank Giuseppe Buttazzo for proposing the problem and for his useful suggestions. The author has been supported by the research group "Problemi di evoluzione nei fluidi e nei solidi”.

\section{REFERENCES}

[BM1] J. M. Ball and V. J. Mizel, Singular minimizers for regular one-dimensional problems in the calculus of variations, Bull. Amer. Math. Soc. (N.S.) 11 (1984), 143-146.

[BM2] __, One-dimensional variational problems whose minimizers do not satisfy the EulerLagrange equation, Arch. Rational Mech. Anal. 90 (1985), 325-388.

[Be] M. Belloni, Ph.D. Thesis (in preparation).

[B] G. Buttazzo, Semicontinuity, relaxation and integral representation in the calculus of variations, Pitman Res. Notes Math. Ser., vol. 203, Longman, Harlow, 1989.

[BuM] G. Buttazzo and V. J. Mizel, Interpretation of the Lavrentiev phenomenon by relaxation, J. Funct. Anal. 110 (1992), 434-460.

[CA] L. Cesari and T. S. Angell, On the Lavrentiev phenomenon, Calcolo 22 (1985), 17-29.

[C] C. W. Cheng, The Lavrentiev phenomenon and its applications in nonlinear elasticity, Ph.D. Thesis, Carnegie Mellon Univ., Pittsburgh, 1993.

[CM] C. W. Cheng and V. J. Mizel, On the Lavrentiev phenomenon for autonomous second order integrands, Arch. Rational Tech. Anal. 126 (1994), 21-34.

[CV] F. H. Clarke and R. B. Vinter, Regularity properties of solutions to the basic problem in the calculus of variations, Trans. Amer. Math. Soc. 291 (1985), 73-98.

[HM1] A. C. Henricher and V. J. Mizel, The Lavrentiev phenomenon for invariant variational problems, Arch. Rational Mech. Anal. 102 (1988), 57-93.

[HM2] _ A new example of the Lavrentiev phenomenon, SIAM J. Control Optim. 26 (1988), 1490-1503.

[L] M. Lavrentiev, Sur quelques problèmes du calcul des variations, Ann. Mat. Pura Appl. 4 (1926), 107-124. 
[M] B. Manià, Sopra un esempio di Lavrentieff, Boll. Un. Mat. Ital. 13 (1934), 146-153.

[MM] M. Marcus and V. J. Mizel, Absolute continuity on tracks and mappings of Sobolev spaces, Arch. Rational Mech. Anal. 45 (1972), 294-320.

Dipartimento di Matematica, Universitá di Parma, Viá Massimo d’Azeglio, 85/A, 63100 PARMA, ITALY

E-mail address: belloni@dm.unipi.it 